\title{
Is hyperprogressive disease a specific phenomenom of immunotherapy?
}

Marta Brambilla ${ }^{1}{ }^{\oplus}$, Giuseppe Lo Russo ${ }^{1}$, Roberto Ferrara ${ }^{1}$, Sara Manglaviti ${ }^{1} \oplus$, Marina Chiara Garassino ${ }^{1}$, Mario Occhipinti ${ }^{1,2}$ (1)

${ }^{1}$ Medical Oncology Department, Fondazione IRCCS Istituto Nazionale Tumori, 20133 Milan, Italy

${ }^{2}$ Medical Oncology Unit B, Policlinico Umberto I "Sapienza" University of Rome, 00161 Rome, Italy

*Correspondence: Mario Occhipinti, Medical Oncology Department, Fondazione IRCCS Istituto Nazionale Tumori, 20133 Milan, Italy; Medical Oncology Unit B, Policlinico Umberto I "Sapienza" University of Rome, 00161 Rome, Italy. mario.occhipinti@ istitutotumori.mi.it

Academic Editor: Rossana Berardi, Università Politecnica Marche, Italy

Received: October 20, 2020 Accepted: December 7, 2020 Published: December 28, 2020

Cite this article: Brambilla M, Lo Russo G, Ferrara R, Manglaviti S, Garassino MC, Occhipinti M. Is hyperprogressive disease a specific phenomenom of immunotherapy. Explor Target Antitumor Ther. 2020;1:427-33. https://doi.org/10.37349/ etat. 2020.00027

\begin{abstract}
Hyperprogressive disease (HPD) is a novel pattern of response during immunotherapy treatment. Several retrospective studies have evaluated its prevalence among various cancer types and, in particular, in nonsmall cell lung cancer patients, based on different definition criteria. If HPD is a just a typical phenomenon of immunotherapy is still an unsolved concern. This paper summarized the available data about HPD in other cancer treatments. Hyperprogressive disease (HPD) is a novel pattern of response during immunotherapy treatment. Several retrospective studies have evaluated its prevalence among various cancer types and, in particular, in non-small cell lung cancer patients, based on different definition criteria. If HPD is a just a typical phenomenon of immunotherapy is still an unsolved concern. This paper summarized the available data about HPD in other cancer treatments.
\end{abstract}

\section{Keywords}

Hyperprogressive disease, immunotherapy, chemotherapy-immunotherapy

\section{Introduction}

The advent of immune checkpoint inhibitors (ICIs) has completely changed the oncology clinical practice producing an increased survival benefit in various tumor types [1-5]. However, the use of ICIs has led to many therapeutic concerns for physicians, mainly due to the reported novel patterns of responses, such as pseudoprogression or hyperprogression. Hyperprogressive disease (HPD) is defined as an unexpected rapid tumor growth occurring in patients treated with immunotherapy. Approximately 3.8-29.4\% of cancer patients and 13.8-37\% of advanced non-small cell lung cancer (NSCLC) patients under anti-programmed cell death $1 /$ programmed death ligand 1 (PD-1/PD-L1) inhibitors, reported HPD [6]. It is still unclear if this phenomenon 
and its definition criteria are specific for ICIs and if HPD has been undervalued with conventional cytotoxic chemotherapy or target therapy.

\section{HPD criteria}

HPD has been defined with different criteria among studies, so understanding the methodological diversity used to classify this phenomenon is essential to better understand the different results and to translate it into clinical practice.

One of the first definition of HPD included tumor growth rate (TGR). The TGR is able to define the kinetics of tumor growth before the start of treatment and its evolution after the beginning of ICIs. TGR is defined as a mathematical equation which considers the percentage of increase in tumor volume in a given time interval [7]. In this experience Champiat et al. [6], compared the tumor growth rate before (TGR B) and tumor growth rate during (TGR D) immunotherapy. HPD was defined as a progressive disease according to response evaluation criteria in solid tumors (RECIST) 1.1. at the first radiological evaluation with a 2-fold increase in the TGR ratio (TGR D/TGR B). The authors reported an inversely proportional correlation between the responses to immunotherapy and the TGR during the treatment period. The same criteria were used by Kanjanapan et al. [8] in their study. Ferrara et al. [9], defined HPD as a progression according to RECIST 1.1. at the first evaluation with an increase of more than $50 \%$ in TGR during ICI (TGR D) compared with TGR before ICI (TGR B) (TGR D-TGR B > 50\%) [9]. Kato et al. [10], used a composite definition of HPD including size-clinical dependent and time dependent criteria: time to treatment failure (TTF) less than 2 months, an increase of more than $50 \%$ of the tumor load and an increase $>2$ of the rate of progression during immunotherapy compared to the pre-immunotherapy period. Matos et al. [11], defined patients with HPD those who had a TTF $<2$ months, a minimum increase in measurable lesions of $10 \mathrm{~mm}$ and an increase of at least $40 \%$ of the tumor burden or an increase of at least $20 \%$ associated with the appearance of new lesions. Saâda-Bouzid et al. [12], used tumor growth kinetics (TGK), a parameter essentially similar to TGR that takes into account the variation of the sum of the larger diameters of the target lesions per unit of time HPD was defined as a tumor growth kinetics ratio (TGKR) $\geq 2$. TGKR is calculated as ratio of the slope of tumor growth pre-immunotherapy and the slope of tumor growth on-treatment. The TGKR does not bring intuitive information related to the difference in volume, since a doubling of the diameter means an increase of 8 times the volume [13]. Finally, in the manuscript by Lo Russo et al. [14], HPD was defined as a progression at the first radiological evaluation according to RECIST 1.1. associated with at least three of the following criteria: TTF $<2$ months, $\geq 50 \%$ increase in the sum of target lesion diameters between baseline and first evaluation; the appearance of at least 2 new lesions in a previously involved organ; the spread of the disease to new organs; worsening of clinical conditions with an increase in PD according to Eastern Cooperative Oncology Group (ECOG) $\geq 2$ during the first 2 months of treatment.

The great difference in the evaluation criteria for HPD reported in the different studies is the likely cause of the different incidences of HPD among cases series [6, 8-12, 14-16] (Table 1).

\section{HPD in other treatment: different characteristics of the same phenomenon?}

HPD has been reported retrospectively in cancer patients under ICIs treatment, however we do not know if it is really a novel pattern of response exclusive of ICIs. Moreover, discontinuation of previous treatments, such as chemotherapy or targeted therapy, may also result in a "disease flare" that may simulate HPD. This phenomenon was defined for the first time by Chaft et al. [17], as a hospitalization or death attributable to disease progression after stopping the EGFR tyrosin kinase inhibitors (TKIs). They found out a disease flare in 14 among the 61 Caucasian patients evaluated (23\%; 95\% CI 14-35\%). Nevertheless, it was not done a comparison of tumor kinetics growth before and after TKI discontinuation due to unavailable data. Chen et al. [18], reported a rate of disease flare of $8 \%$ in Asian patients after the cessation of EGFR-TKIs. Similar data were found also for patients discontinuing ALK-tyrosine kinase inhibitors or VEGFR-tyrosine kinase inhibitors $[19,20]$. Moreover, cancer flare has been described for bone disease progression in NSCLC patients under EGFR-TKIs as an effect of an increased osteoblastic and healing activity and sign of therapeutic 
effect [21]. Acceleration of tumor growth during targeted agents (TAs) treatment has been described for RAF inhibitors and BRAF inhibitors [22-26]. Recently Matos et al. [27], evaluated the range of HPD in a cohort of advanced solid tumors treated in phase I trials with TAs in unapproved indications. From 119 patients who had progressive disease as best response, 26 (21.8\%) were classified as HPD by RECIST criteria. No differences in overall survival (OS) were observed between HPD group (median OS 4.23 months; 95\% CI 3.42-5.04) and non-HPD progressor group (median OS 5.7; 95\% CI 4.99-6.4; HR 1.09, 95\% CI 0.7-1.7; $P=0.70$ ). This study has several limitations. It is a retrospective evaluation in which TAs, already approved for a clinical indication, were excluded; moreover, were included several tumor types and different kinds of target treatments and all patients were at umpteenth lines underlaying an intrinsic tumor resistance and aggressiveness. At least the use of RECIST 1.1 criteria may have overestimated HPD phenomenon in tumors with an aggressive intrinsic biology and underestimated it in patients with a rapid clinical deterioration without a confirmatory scan.

Table 1. List of the main criteria for HPD definition

\begin{tabular}{|c|c|c|c|c|c|c|}
\hline HPD definition & Drug & $\begin{array}{l}\text { Type of } \\
\text { tumor }\end{array}$ & $\begin{array}{l}\text { Patients } \\
(N)\end{array}$ & HPD (\%) & $\begin{array}{l}\text { Predictive } \\
\text { factors }\end{array}$ & Reference \\
\hline $\begin{array}{l}\text { PD RECIST and increase in } \\
T G R \geq 2\end{array}$ & $\begin{array}{l}\text { mAb anti PD-1/PD-L1 } \\
\text { (phase I) }\end{array}$ & Various & 131 & $9 \%$ & Age $\geq 65$ years & {$[6]$} \\
\hline PD RECIST and $\Delta \mathrm{TGR}>50 \%$ & mAb anti PD-1/PD-L1 & NSCLC & 406 & $13.8 \%$ & $\begin{array}{l}>2 \text { metastatic } \\
\text { sites }\end{array}$ & [9] \\
\hline $\begin{array}{l}\text { PD RECIST \& increase in TGR } \\
\geq 2\end{array}$ & $\begin{array}{l}\text { CKI and costimulatory } \\
\text { molecules }\end{array}$ & Various & 182 & $7 \%$ & Female & {$[8]$} \\
\hline Increase in TGK $\geq 2$ & $\begin{array}{l}\text { mAb anti } \\
\text { PD-1/PD-L1 }\end{array}$ & HNSCC & 34 & $29 \%$ & $\begin{array}{l}\text { Tumor relapse } \\
\text { in } \mathrm{RT}\end{array}$ & [12] \\
\hline $\begin{array}{l}\text { PD RECIST and } 3 \text { criteria } \\
\text { among: } \\
\text { 1) TTF }<2 \text { months; } \\
\text { 2) increase } \geq 50 \% \text { in sum of } \\
\text { target diameters; } \\
3 \text { ) } \geq 2 \text { new lesions in already } \\
\text { involved organs; } \\
\text { 4) Mets in new organs; } \\
\text { 5) PS ECOG } \geq 2\end{array}$ & CKI & NSCLC & 152 & $25.7 \%$ & $\begin{array}{l}\text { Myeloids cell } \\
\text { density and MPO } \\
\text { in the tumor \& } \\
\text { low PD-L1 }\end{array}$ & {$[14]$} \\
\hline $\begin{array}{l}\text { TTF }<2 \text { months and increase } \\
\text { in tumor burden } \geq 50 \% \text { and } \\
\text { increase in pace of growth } \geq 2\end{array}$ & $\begin{array}{l}\text { CKI and costimulatory } \\
\text { molecules }\end{array}$ & Various & 155 & $4 \%$ & $\begin{array}{l}\text { EGFR, MDM2/4, } \\
\text { DNMT3A }\end{array}$ & [10] \\
\hline $\begin{array}{l}\text { TTF }<2 \text { months and increase } \\
\geq 10 \mathrm{~mm} \text { in mesurable lesions } \\
\text { and increase } \geq 40 \% \text { of } \\
\text { tumor burden or }>20 \% \text { with } \\
\text { apperance of new lesions }\end{array}$ & CKI & Various & 214 & $15 \%$ & & [11] \\
\hline $\begin{array}{l}\mathrm{TTF}<2 \text { months and TGK } \geq 2 \\
\text { and increase in tumor volume } \\
\geq 50 \%\end{array}$ & CKI & NSCLC & 135 & $13.1 \%$ & $\begin{array}{l}\text { NLR > 4, LDH > } \\
\text { UNL, STK11 }\end{array}$ & [15] \\
\hline $\begin{array}{l}\text { PD RECIST and RECIST } \\
\geq 50 \% \text { and increase in TGR } \geq 2\end{array}$ & CKI & Various & & $\begin{array}{l}5 \mathrm{HPD} \\
\text { patients }\end{array}$ & MDM2/4, EGFR & [16] \\
\hline
\end{tabular}

PD: progressive disease; PS: performance status; mAb: monoclonal antibody; CKI: cyclin-dependent kinase inhibitors; HNSCC: head and neck squamous cell carcinoma; RT: radiotherapy; MPO: myeloperoxidase; MDM2/4: Mouse double minute 2/4 homolog; DNMT3A: DNA methyltransferase 3 alpha; NLR: neutrophil lymphocyte ratio; LDH: lactate dehydrogenase; UNL: upper normal limit; STK11: Serine/threonine kinase 11

A rapid tumour cell proliferation was seen after induction chemotherapy in oropharyngeal cancer [28]. Nevertheless, to date there is only one study that evaluated the incidence of HPD between NSCLC patients under single agent chemotherapy. Using delta TGR Ferrara et al. [9], reported, in the 59 NSCLC patients included in their analysis only 3 HPD cases, with a median OS of 4.5 months (95\% CI 2.5-6.5 months) at the landmark analysis at 6 weeks. Therefore, HPD was observed in 13.8\% (56 of 406) of patients treated with PD-1/PD-L1 inhibitors compared with 5.1\% (3 of 59) of patients treated with single-agent chemotherapy (taxanes 73\%, pemetrexed 12\%, vinorelbine 7\%, and gemcitabine $8 \%$ ). This study underlines that HPD is clearly more frequent in patients treated with immunotherapy but maybe not exclusive of these patients. 
Even less is known about HPD presence in first line treatment with chemotherapy-immunotherapy. The efficacy of pembrolizumab in combination treatment was tested in two phase III studies in both squamous and non-squamous histotypes, respectively KEYNOTE 407 [29] and KEYNOTE 189 [30]. In the squamous histotype the addition of pembrolizumab to a platinum-based chemotherapy (associated with paclitaxel or nab-paclitaxel) has demonstrated to have a significant benefit over chemotherapy alone in progression free survival (PFS) and OS, regardless of PD-L1 status. Similarly, in patients with non-squamous histotype, the addition of pembrolizumab to a platinum and pemetrexed doublet showed a benefit in both OS and PFS compared to chemotherapy alone. In both studies the experimental treatment was not associated with an increased incidence of side effects or an accelerate progression of the disease. Even atezolizumab was evaluated in combination with first-line chemotherapy in patients with advanced lung cancer. In nonsquamous histology, the association of atezolizumab has been evaluated in 3 phase III randomized trials: IMpower 150, 130 and 132 [31-33], respectively evaluating the addition of atezolizumab to a carboplatinpaclitaxel-bevacizumab, platinum salts-nab-paclitaxel, platinum salts-pemetrexed. All these studies showed a statistically significant advantage in their endpoints with the addition of immunotherapy to chemotherapy and no evidence of early deleterious effect in the combination arm. On the contrary, in squamous histology the addition of atezolizumab to a platinum-based doublet was evaluated in the IMpower 131 study and has not demonstrated a statistically significant benefit in terms of survival [33]. However, even in this study no detrimental effect was observed compared to chemotherapy alone.

Thus, understanding whether chemotherapy-immunotherapy may prevent HPD effect of ICI is still an unsolved question. Moreover, it is still unclear if the criteria used for single agent immunotherapy may be applied also in this setting. A recent report presented at the European Society for Medical Oncology (ESMO) 2020 compared the incidence of HPD between single agent ICI vs. combination therapy as front-line treatment in NSCLC. HPD occurred in up to $16 \%$ of PD-L1 TPS $\geq 50 \%$ NSCLC patients treated with first-line singleagent ICI compared to $6 \%$ in chemotherapy-immunotherapy. Furthermore, none of the HPD reported in the combination treatment was detected by dynamic indexes (TGR/TGK), suggesting that clinic-radiological features of HPD in this chemotherapy-immunotherapy may be different from the ICI single agent ones [34].

\section{Conclusions}

The phenomenon of HPD has been highlighted with the advent of ICIs treatment. A possible explanation of HPD in patients treated with ICIs could lay in the enhancement of pro-tumorigenic effect of immune-system, such a switch to immunesuppressive tumor microenvironment by a stimulation of regulatory $\mathrm{T}$ cells via PDL1 blockade [6]. Other possible mechanisms underlying HPD could be linked to tumor-infiltration by M2-like $\mathrm{CD}_{163}{ }^{+} \mathrm{CD} 33^{+} \mathrm{PD}-\mathrm{L} 1^{+}$clustered epithelioid macrophages [14] or to specific gene expression signatures [35].

Chemotherapy-immunotherapies treatment seems to lower the risk of experience HPD, suggesting that the use of combination treatments not inciting to immune-mechanisms may be a potential strategy to overcome HPD and maximize ICIs benefit. We have too little data to express an opinion regarding the possible presence of the HPD phenomenon in patients treated with chemotherapy or targeted therapy. Therefore, we need prospective studies to validate the hypotheses and to better understand HPD phenomenon.

\section{Abbreviations}

ECOG: Eastern Cooperative Oncology Group

HPD: hyperprogressive disease

ICIs: immune checkpoint inhibitors

NSCLC: non-small cell lung cancer

OS: overall survival

PD-1: programmed cell death 1

PD-L1: programmed death ligand 1

PFS: progression free survival 
RECIST: response evaluation criteria in solid tumors

TAs: targeted agents

TGK: tumor growth kinetics

TGKR: tumor growth kinetics ratio

TGR: tumor growth rate

TGR B: tumor growth rate before

TGR D: tumor growth rate during

TKIs: tyrosin kinase inhibitors

TTF: time to treatment failure

\section{Declarations}

\section{Author contributions}

MB, MO and SM contributed conception and writing of the manuscript; GLR, RF and MCG contributed to manuscript revision. All authors read and approved the submitted version.

\section{Conflict of interests}

MCG declares personal financial interests with the following organizations: AstraZeneca, MSD International GmbH, BMS, Boehringer Ingelheim Italia S.p.A, Celgene, Eli Lilly, Ignyta, Incyte, Inivata, MedImmune, Novartis, Pfizer, Roche, Takeda; she also declares Institutional financial interests with the following organizations: Eli Lilly, MSD, Pfizer (MISP), AstraZeneca, MSD International GmbH, BMS, Boehringer Ingelheim Italia S.p.A, Celgene, Ignyta, Incyte, Inivata, MedImmune, Novartis, Pfizer, Roche, Takeda, Tiziana, Foundation Medicine; at the end, she has received research funding from the following organizations: AIRC, AIFA, Italian Moh, TRANSCAN, outside the submitted work. GLR declares personal fees from Eli Lilly, BMS and AstraZeneca, outside the submitted work. The other authors declare that they have no conflicts of interest.

\section{Ethical approval}

Not applicable.

\section{Consent to participate}

Not applicable.

\section{Consent to publication}

Not applicable.

Availability of data and materials

Not applicable.

\section{Funding}

Not applicable.

\section{Copyright}

(C) The Authors 2020.

\section{References}

1. Schachter J, Ribas A, Long GV, Arance A, Grob JJ, Mortier L, et al. Pembrolizumab versus ipilimumab for advanced melanoma: final overall survival results of a multicentre, randomised, open-label phase 3 study (KEYNOTE-006). Lancet. 2017;390:1853-62. 
2. Brahmer J, Reckamp KL, Baas P, Crinò L, Eberhardt WEE, Poddubskaya E, et al. Nivolumab versus docetaxel in advanced squamous-cell non-small-cell lung cancer. N Engl J Med. 2015;373:123-35.

3. Escudier B, Sharma P, McDermott DF, George S, Hammers HJ, Srinivas S, et al. CheckMate 025 randomized phase 3 study: outcomes by key baseline factors and prior therapy for nivolumab versus everolimus in advanced renal cell carcinoma. Eur Urol. 2017;72:962-71.

4. Ferris RL, Blumenschein G Jr, Fayette J, Guigay J, Colevas AD, Licitra L, et al. Nivolumab for recurrent squamous-cell carcinoma of the head and neck. N Engl J Med. 2016;375:1856-67.

5. Wolchok JD, Hoos A, O'Day S, Weber JS, Hamid O, Lebbé C, et al. Guidelines for the evaluation of immune therapy activity in solid tumors: immune-related response criteria. Clin Cancer Res. 2009;15:7412-20.

6. Champiat S, Dercle L, Ammari S, Massard C, Hollebecque A, Postel-Vinay S, et al. Hyperprogressive disease is a new pattern of progression in cancer patients treated by anti-PD-1/PD-L1. Clin Cancer Res. 2017;23:1920-8.

7. Ferté C, Fernandez M, Hollebecque A, Koscielny S, Levy A, Massard C, et al. Tumor growth rate is an early indicator of antitumor drug activity in phase I clinical trials. Clin Cancer Res. 2014;20:246-52.

8. Kanjanapan Y, Day D, Wang L, Al-Sawaihey H, Abbas E, Namini A, et al. Hyperprogressive disease in earlyphase immunotherapy trials: clinical predictors and association with immune- related toxicities. Cancer. 2019;125:1341-9.

9. Ferrara R, Mezquita L, Texier M, Lahmar J, Audigier-Valette C, Tessonnier L, et al. Hyperprogressive disease in patients with advanced non-small cell lung cancer treated with PD-1/PD-L1 inhibitors or with single-agent chemotherapy. JAMA Oncol. 2018;4:1543-52.

10. Kato S, Goodman A, Walavalkar V, Barkauskas DA, Sharabi A, Kurzrock R. Hyperprogressors after immunotherapy: analysis of genomic alterations associated with accelerated growth rate. Clin Cancer Res. 2017;23:4242-50.

11. Matos I, Martin-Liberal J, Hierro C, Olza MO, Viaplana C, Costa M, et al. Incidence and clinical implications of a new definition of hyperprogression (HPD) with immune checkpoint inhibitors (ICIs) in patients treated in phase 1 (Ph1) trials. J Clin Oncol. 2018;36 Suppl 15:3032.

12. Saâda-Bouzid E, Defaucheux C, Karabajakian A, Coloma VP, Servois V, Paoletti X, et al. Hyperprogression during anti-PD-1/PD-L1 therapy in patients with recurrent and/or metastatic head and neck squamous cell carcinoma. Ann Oncol. 2017;28:1605-11.

13. Le Tourneau C, Servois V, Diéras V, Ollivier L, Tresca P, Paoletti X. Tumour growth kinetics assessment: added value to RECIST in cancer patients treated with molecularly targeted agents. $\mathrm{Br} \mathrm{J}$ Cancer. 2012;106:854-7.

14. Lo Russo G, Moro M, Sommariva M, Cancila V, Boeri M, Centonze G, et al. Antibody-Fc/FcR interaction on macrophages as a mechanism for hyperprogressive disease in non-small cell lung cancer subsequent to PD-1/PD-L1 blockade. Clin Cancer Res. 2019;25:989-99.

15. Kim Y, Kim CH, Kim HS, Sun J, Ahn JS, Ahn M, et al. Hyperprogression after immunotherapy: clinical implication and genomic alterations in advanced non-small cell lung cancer patients (NSCLC). J Clin Oncol. 2018;36 Suppl 15:9075.

16. Singavi AK, Menon S, Kilari D, Alqwasmi A, Ritch PS, Thomas JP, et al. Predictive biomarkers for hyperprogression (HP) in response to immune checkpoint inhibitors (ICI)-analysis of somatic alterations (SAs). Ann Oncol. 2017;28 Suppl 5:V405.

17. Chaft JE, Oxnard GR, Sima CS, Kris MG, Miller VA, Riely GJ. Disease flare after tyrosine kinase inhibitor discontinuation in patients with EGFR-mutant lung cancer and acquired resistance to erlotinib or gefitinib: implications for clinical trial design. Clin Cancer Res. 2011;17:6298-303.

18. Chen HJ, Yan HH, Yang JJ, Chen ZH, Su J, Zhang XC, et al. Disease flare after EGFR tyrosine kinase inhibitor cessation predicts poor survival in patients with non-small cell lung cancer. Pathol Oncol Res. 2013;19:833-8. 
19. Kuriyama Y, Kim YH, Nagai H, Ozasa H, Sakamori Y, Mishima M. Disease flare after discontinuation of crizotinib in anaplastic lymphoma kinase-positive lung cancer. Case Rep Oncol. 2013;6:430-3.

20. Iacovelli R, Massari F, Albiges L, Loriot Y, Massard C, Fizazi K, et al. Evidence and clinical relevance of tumor flare in patients who discontinue tyrosine kinase inhibitors for treatment of metastatic renal cell carcinoma. Eur Urol. 2015;68:154-60.

21. de Bondt C, Snoeckx A, Raskin J. Correction: A flare for the unexpected: bone flare as response to tyrosine kinase inhibitor treatment in a lung cancer patient. J Belg Soc Radiol. 2020;104:28. Erratum for: J Belg Soc Radiol. 2020;104:18.

22. Mellema WW, Masen-Poos L, Smit EF, Hendriks LE, Aerts JG, Termeer A, et al. Comparison of clinical outcome after first-line platinum-based chemotherapy in different types of KRAS mutated advanced non-small-cell lung cancer. Lung Cancer. 2015;90:249-54.

23. Heidorn SJ, Milagre C, Whittaker S, Nourry A, Niculescu-Duvas I, Dhomen N, et al. Kinase-dead BRAF and oncogenic RAS cooperate to drive tumor progression through CRAF. Cell. 2010;140:209-21.

24. Su F, Viros A, Milagre C, Trunzer K, Bollag G, Spleiss O, et al. RAS mutations in cutaneous squamous-cell carcinomas in patients treated with BRAF inhibitors. N Engl J Med. 2012;366:207-15.

25. Andrews MC, Behren A, Chionh F, Mariadason J, Vella LJ, Do H, et al. BRAF inhibitor-driven tumor proliferation in a KRAS-mutated colon carcinoma is not overcome by MEK1/2 inhibition. J Clin Oncol. 2013;31:e448-51.

26. Boussemart L, Girault I, Malka-Mahieu H, Mateus C, Routier E, Rubington M, et al. Secondary tumors arising in patients undergoing BRAF inhibitor therapy exhibit increased BRAF-CRAF heterodimerization. Cancer Res. 2016;76:1476-84.

27. Matos I, Martin-Liberal J, García-Ruiz A, Hierro C, De Olza MO, Viaplana C, etal. Capturing hyperprogressive disease with immune-checkpoint inhibitors using RECIST 1.1 criteria. Clin Cancer Res. 2020;26:1846-55.

28. Bourhis J, Wilson G, Wibault P, Janot F, Bosq J, Armand JP, et al. Rapid tumor cell proliferation after induction chemotherapy in oropharyngeal cancer. Laryngoscope. 1994;104:468-72.

29. Paz-Ares L, Luft A, Vicente D, Tafreshi A, Gümüş M, Mazières J, et al. Pembrolizumab plus chemotherapy for squamous non-small-cell lung cancer. N Engl J Med. 2018;379:2040-51.

30. Gandhi L, Rodríguez-Abreu D, Gadgeel S, Esteban E, Felip E, De Angelis F, et al. Pembrolizumab plus chemotherapy in metastatic non-small-cell lung cancer. N Engl J Med. 2018;378:2078-92.

31. Barlesi F, Nishio M, Cobo M, Steele N, Paramonov V, Parente B, et al. IMpower132: efficacy of atezolizumab (atezo) + carboplatin (carbo)/cisplatin (cis) + pemetrexed (pem) as 1L treatment in key subgroups with stage IV non-squamous non-small cell lung cancer (NSCLC). Ann Oncol. 2018;29 Suppl 8:viii743-4.

32. West H, McCleod M, Hussein M, Morabito A, Rittmeyer A, Conter HJ, et al. Atezolizumab in combination with carboplatin plus nab-paclitaxel chemotherapy compared with chemotherapy alone as first-line treatment for metastatic non-squamous non-small-cell lung cancer (IMpower130): a multicentre, randomised, open-label, phase 3 tria. Lancet Oncol. 2019;20:924-37.

33. Socinski MA, Jotte RM, Cappuzzo F, Orlandi F, Stroyakovskiy D, Nogami N, et al. Atezolizumab for firstline treatment of metastatic nonsquamous NSCLC. N Engl J Med. 2018;378:2288-301.

34. Ferrara R, Facchinetti F, Calareso G, Kasraoui I, Signorelli D, Proto C, et al. Hyperprogressive disease upon first-line PD-1/PD-L1 inhibitors (ICI) as single agent or in combination with platinum-based chemotherapy in non-small cell lung cancer (NSCLC) patients (pts). Ann Oncol. 2020;31 Suppl 4:S826.

35. Xiong D, Wang Y, Singavi AK, Mackinnon AC, George B, You M. Immunogenomic landscape contributes to hyperprogressive disease after anti-PD-1 immunotherapy for cancer. iScience. 2018;9:258-77. 